\title{
Reduction of thermal conductivity in ferroelectric $\mathrm{SrTiO}_{3}$ thin films
}

\author{
Alexandros Sarantopoulos $\odot,{ }^{1,2, *}$ Dipanjan Saha $\odot,{ }^{3}$ Wee-Liat Ong, ${ }^{3,4,5}$ César Magén $\odot,{ }^{6,7}$ \\ Jonathan A. Malen, ${ }^{3, \dagger}$ and Francisco Rivadulla ${ }^{1}{ }^{1}$ \\ ${ }^{1}$ Centro de Investigación en Química Biológica y Materiales Moleculares (CIQUS), Universidad de Santiago de Compostela, \\ 15782 Santiago de Compostela, Spain \\ ${ }^{2}$ Peter Gruenberg Institute (PGI-7), Forschungszentrum Juelich GmbH and JARA-FIT, 52425 Juelich, Germany \\ ${ }^{3}$ Department of Mechanical Engineering, Carnegie Mellon University, Pittsburgh, Pennsylvania 15213, USA \\ ${ }^{4}$ ZJU-UIUC Institute, College of Energy Engineering, Zhejiang University, Hangzhou, Zhejiang 314400, China \\ ${ }^{5}$ State Key Laboratory of Clean Energy Utilization, Zhejiang University, Hangzhou, Zhejiang 310027, China \\ ${ }^{6}$ Instituto de Ciencia de Materiales de Aragón (ICMA), Universidad de Zaragoza-CSIC, and Departamento de Física de la Materia \\ Condensada, Universidad de Zaragoza, 50009 Zaragoza, Spain \\ ${ }^{7}$ Laboratorio de Microscopías Avanzadas - Instituto de Nanociencia de Aragón, Universidad de Zaragoza, 50018 Zaragoza, Spain
}

(Received 19 November 2019; revised manuscript received 27 March 2020; accepted 16 April 2020; published 14 May 2020)

\begin{abstract}
Bulk $\mathrm{SrTiO}_{3}$ is a quantum paraelectric in which an antiferrodistortive distortion below $\approx 105 \mathrm{~K}$ and quantum fluctuations at low temperature preclude the stabilization of a long-range ferroelectric state. However, biaxial mechanical stress, impurity doping, and Sr nonstoichiometry, among other mechanisms, are able to stabilize a ferroelectric or relaxor ferroelectric state at room temperature, which develops into a longer-range ferroelectric state below $250 \mathrm{~K}$. In this paper, we show that epitaxial $\mathrm{SrTiO}_{3}$ thin films grown under tensile strain on $\mathrm{DyScO}_{3}$ exhibit a large reduction of thermal conductivity, of $\approx 60 \%$ at room temperature, with respect to identical strain-free or compressed films. The thermal conductivity shows a further reduction below $250 \mathrm{~K}$, a temperature concurrent with the peak in the dielectric constant [J. H. Haeni et al., Nature (London) 430, 758 (2004)]. These results suggest that strain gradients in the relaxor and ferroelectric phase of $\mathrm{SrTiO}_{3}$ are very effective phonon scatterers, limiting the thermal transport in this material.
\end{abstract}

DOI: 10.1103/PhysRevMaterials.4.054002

\section{INTRODUCTION}

Stoichiometric $\mathrm{SrTiO}_{3}$ (STO) undergoes an antiferrodistortive structural transition at $105 \mathrm{~K}$, from cubic to tetragonal, that competes with ferroelectricity [1,2]. This competition can be biased by external pressure or a sufficiently large epitaxial strain [3]. Following this approach, Haeni et al. [4] stabilized a ferroelectric state at room temperature in thin films of STO under $\approx 1.1 \%$ biaxial tensile strain, creating a large in-plane electric polarization with a complex domain structure [5]. Temperature-dependent spectroscopic experiments pointed towards the development of a full ferroelectric state below $270 \mathrm{~K}$ in this system, driven by a soft-phonon instability [6]. Independent measurements confirmed the maximum in the dielectric constant at $\approx 250 \mathrm{~K} \mathrm{[7]}$, and highlighted the critical role played by defects in stabilizing a relaxor-ferroelectric behavior in strained STO thin films [8]. Actually, Jang et al. [9] proposed that nanopolar regions linked to unintentional $\mathrm{Sr}^{2+}$ vacancies in STO result in a relaxor ferroelectric state at room temperature. In this scenario, tensile epitaxial strain will induce long-range correlation between these preexisting polar domains and stabilize a longer-range ferroelectric state at low temperature.

\footnotetext{
*a.sarantopoulos@fz-juelich.de

†jonmalen@andrew.cmu.edu
}

The presence of domains with electrical polarization pointing along different directions will riddle the material with polarization and strain gradients extending along ferroelectric domain walls (FEDWs), which in turn are proposed to be effective phonon scatterers [10-16]. In this regard, strained STO offers a unique model system to quantify the effect of FEDWs on the thermal conductivity $\kappa(T)$.

Here, we report a large reduction of $\kappa$ at room temperature for epitaxial thin films of STO under tensile strain, and a further suppression below $\approx 250 \mathrm{~K}$, suggesting the complex transition to a ferroelectric state in this system. Our results thus support the argument of the ability of FEDWs to control $\kappa$ of ferroelectric materials.

\section{SAMPLE PREPARATION}

Epitaxial thin films of STO were grown by pulsed laser deposition (PLD) $(\mathrm{KrF}, \lambda=248 \mathrm{~nm}$, and $\mathrm{Nd}: \mathrm{YAG}, \lambda=266 \mathrm{~nm}$, laser fluence $0.9 \mathrm{~J} / \mathrm{cm}^{2}, 5 \mathrm{~Hz}$, at $800^{\circ} \mathrm{C}$ ) on single-crystal (001) STO $(a=3.905 \AA)$, (001) $\left(\mathrm{LaAlO}_{3}\right)_{0.3}\left(\mathrm{Sr}_{2} \mathrm{AlTaO}_{6}\right)_{0.7}$ (LSAT, $a=3.87 \AA$ ), and (110) orthorhombic $\mathrm{DyScO}_{3}$ (DSO, $a_{p c}=3.95 \AA$ ) substrates. These have been selected to induce an epitaxial strain in STO from $\approx-0.9 \%$ in LSAT, to $\approx+1.15 \%$ on DSO. The oxygen pressure inside the PLD chamber was $0.1-10^{-3}$ Torr, depending on the sample. The substrates were thermally treated, before deposition, to achieve atomically flat surfaces [17]. As a source material for 

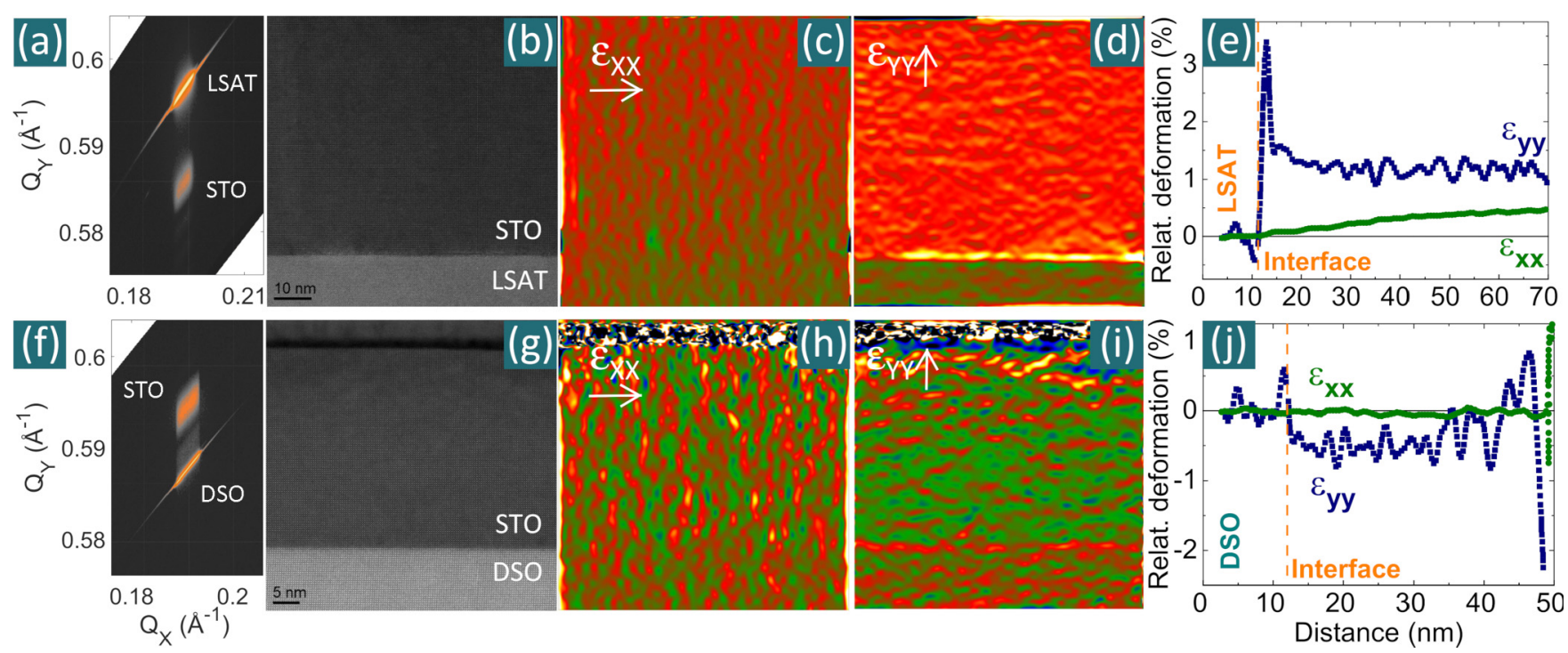

FIG. 1. High-resolution reciprocal space maps (RSMs) of STO thin films grown on (a) LSAT (65 nm) and (f) DSO (50 nm) substrates at 100 mTorr, around the asymmetric Bragg reflections (103) and (332), respectively. Cross-section high-resolution HAADF-STEM images of STO thin films grown on (b) LSAT and (g) DSO, along with their corresponding in-plane, $\varepsilon_{x x}\left[(\mathrm{c})\right.$ and (h)], and out-of-plane, $\varepsilon_{y y}$ [(d) and (i)], strain maps obtained from a geometrical phase analysis of the HAADF-STEM images. The line profiles of $\varepsilon_{x x}$ and $\varepsilon_{y y}$ along the film thickness, for the films on LSAT and DSO substrates, are plotted in (e) and (j), respectively.

the depositions, a ceramic target was used whose stoichiometric composition was verified by energy-dispersive $\mathrm{x}$-ray spectroscopy (EDX) analysis. The ceramic target was ball milled and sintered at different temperatures three different times to achieve the optimal density.

\section{STRUCTURAL CHARACTERIZATION}

A $\theta / 2 \theta$ x-ray diffraction and reciprocal space map (RSM) analysis shows good matching between the in-plane lattice parameter of the STO films and the different substrates; see Figs. 1(a) and 1(f). In response to epitaxial strain, the films expand/compress in the out-of-plane direction.

The crystalline quality and the distribution of strain along the film's thickness were studied on selected crosssectional specimens by the geometrical phase analysis (GPA) of high-angle annular dark field (HAADF) images in scanning transmission electron microscopy (STEM). The compressive/tensile strain results in a homogeneous elongation/contraction of the out-of-plane lattice parameter, as shown by line profiles of $\varepsilon_{x x}$ and $\varepsilon_{y y}$ in Figs. 1(e) and 1(j). Only a minor variation over the film thickness is observed in the in-plane deformation of the STO grown on the LSAT substrate. Therefore, the STO thin films have undergone a uniform tetragonal deformation, without notable indications of microstructural relaxation.

\section{THERMAL CONDUCTIVITY MEASUREMENTS \& ANALYSIS METHODS}

The thermal conductivities of the films were measured by frequency domain thermoreflectance (FDTR) [18,19]. A sinusoidally modulated continuous wave (cw) pump laser ( $\lambda=488 \mathrm{~nm}$, modulating $f=0.1-6 \mathrm{MHz}$ ) is focused on the surface of the film, coated by a 70-nm-thick layer of sputtered $\mathrm{Au}$, to produce an oscillatory modulation of the surface temperature. This, in turn, results in a periodic change of the Au thermoreflectance, which is subsequently measured by a cw probe laser beam $(\lambda=532 \mathrm{~nm})$. From the phase lag between the pump and the probe laser beams, the effective thermal conductivity of the thin film, $\kappa_{\text {eff }}$, can be determined. If the characteristic time for heat diffusion in the sample $\left(t^{2} / \alpha\right.$, where $t$ and $\alpha$ are the film thickness and thermal diffusivity, respectively) is much shorter than the timescale of the heat modulation $\left(f^{-1}\right)$, the temperature profile in the film can be approximated by a steady-state one-dimensional (1D) solution along its thickness. In this limit, the contribution of the "intrinsic" thermal resistance of the film and the different interfaces can be added to obtain the total thermal resistance,

$$
\frac{t}{\kappa_{\text {eff }}}=\frac{t}{\kappa}+R_{\text {int }},
$$

where $\kappa$ is the "intrinsic" thermal conductivity of the film and $R_{\text {int }}$ is the total sum of the thermal resistance due to the interfaces. Thus, the "intrinsic" thermal conductivity of every film can be obtained from the slope of $t / \kappa_{\mathrm{eff}}$ vs $t$, measured for a series of samples of different thicknesses as long as the film's thermal conductivity is not thickness dependent. We grew four different films with thicknesses between 10 and $65 \mathrm{~nm}$ on each substrate under identical conditions. The good fitting to Eq. (1) [shown in Fig. 2(a)] validates the steady-state 1D approximation, and justifies the validity of the "intrinsic" thermal conductivity. This procedure was repeated for every temperature analyzed in this paper. Note that increasing the film thickness also increases the grain size in polycrystalline films, and, in the case of ferroelectrics, also the FE domain size [20-22]. If domain sizes are comparable to phonon mean free paths and their changes with thickness are significant, $\kappa$ itself may increase with thickness. Increases in $\kappa$ with 

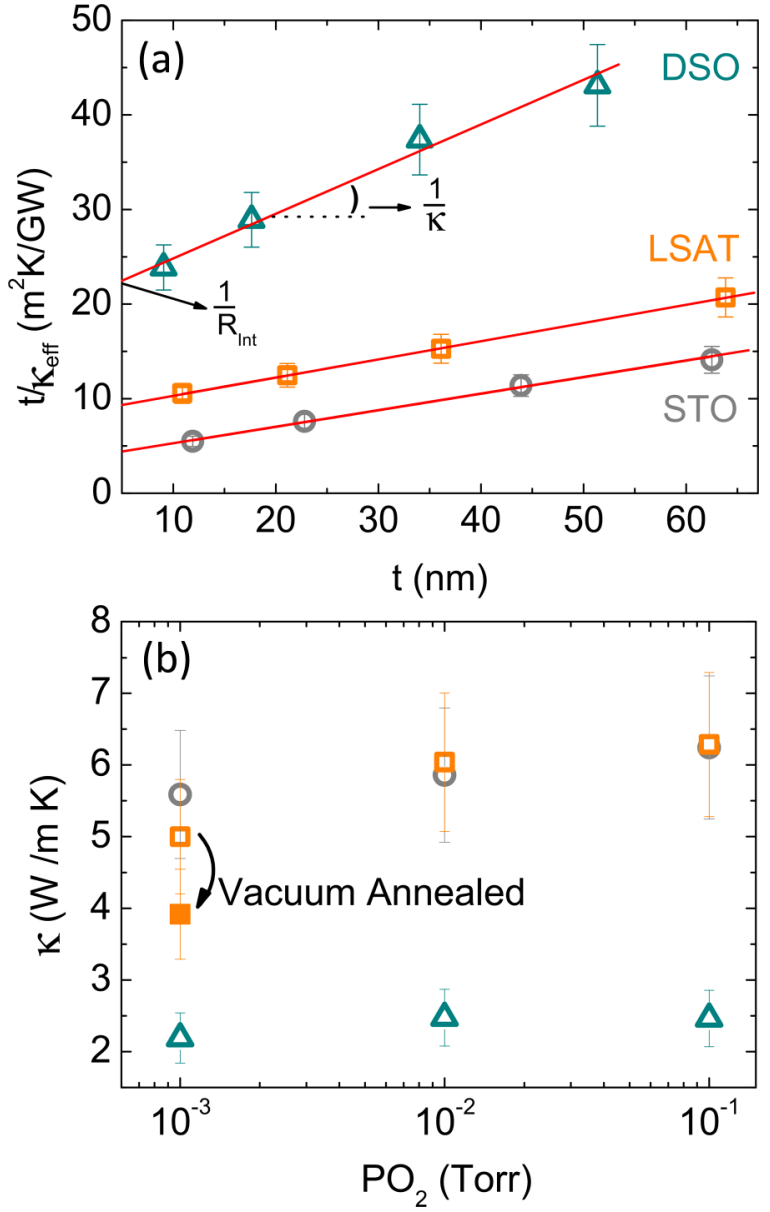

FIG. 2. (a) Thickness dependence of the effective thermal conductivity, according to Eq. (1). The data correspond to a set of films deposited on LSAT (squares) at $\mathrm{PO}_{2}=0.1$ Torr, STO (circles) at $\mathrm{PO}_{2}=10^{-2}$ Torr, and DSO (triangles) at $\mathrm{PO}_{2}=10^{-3}$ Torr, substrates. (b) Room-temperature "intrinsic" $\kappa$ of the STO thin films deposited on top of LSAT, STO, and DSO substrates at different oxygen pressures.

thickness would register as nonlinear, concave down $t / \kappa_{\text {eff }}$ vs $t$, which we do not observe (see Fig. S03 in the Supplemental Material [23]).

\section{RESULTS \& DISCUSSION}

The results show that at room temperature, $\kappa \approx$ $2.5 \mathrm{~W} \mathrm{~m}^{-1} \mathrm{~K}^{-1}$ for STO on DSO, which is $\approx 60 \%$ lower than $\approx 6 \mathrm{~W} \mathrm{~m}^{-1} \mathrm{~K}^{-1}$ measured for STO on LSAT and STO.

To probe any possible contribution from an anomalously large concentration of vacancies on the STO on DSO films [24,25], a series of samples was deposited at lower oxygen pressures, down to $10^{-3}$ Torr. Reducing $\mathrm{PO}_{2}$ during deposition increases the $c$-axis expansion, due to an increasing concentration of point defects (mostly $\mathrm{Sr}^{2+}$ and $\mathrm{O}^{2-}$ vacancies) [24,26-30].

However, as can be seen in Fig. 2(b), the density of vacancies generated in this range of pressure has only a moderate impact in $\kappa$. We also performed a postdeposition annealing at $600{ }^{\circ} \mathrm{C}$ at $10^{-6}$ Torr. The number of generated $V_{\mathrm{O}}$ was

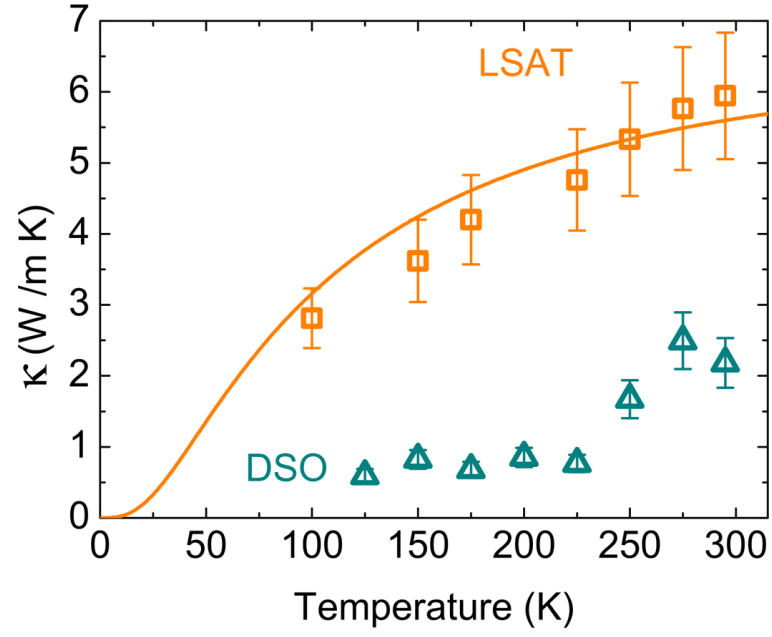

FIG. 3. Temperature-dependent thermal conductivity for STO thin films deposited on top of LSAT and DSO at $\mathrm{PO}_{2}=0.1$ Torr. The solid line correspond to the fitting to the modified Callaway model for STO on LSAT.

estimated $\approx 2 \%$ from the charge carrier density determined by Hall effect measurements, assuming that each $V_{\mathrm{O}}$ donates two electrons to the conduction band of STO [28]. The thermal conductivity was reduced $\approx 25 \%$ after annealing [see Fig. 2(b)]; therefore, the $\approx 60 \%$ reduction of $\kappa$ observed for STO grown on DSO seems too large to be compatible with the effect of point defects.

The change in the total unit-cell volume is also too small to account for the large reduction of $\kappa$. For instance, $\approx+1.15 \%$ in-plane enlargement and the corresponding out-of-plane contraction observed from $x$-ray diffraction resulted in an increase of the volume of STO of $\approx+2 \%$. This causes a change in the Debye temperature $\theta_{D}$ and therefore in $\kappa$, which can be estimated through the Grüneisen parameter $\gamma=-\frac{d \ln \theta_{D}}{d \ln V}$. Taking $\gamma=1.5$ [31], the measured volume change will reduce $\theta_{D}$ by $\approx 3 \%$, leading to an overall reduction of $\kappa$ by $\approx 9 \%(\kappa \propto$ $\left.\theta_{D}^{3}\right)$, again too small to explain the experimental observations.

To gain further insight on the origin of the reduced $\kappa$ observed in tensile strained STO thin films, we measure its temperature dependence from 100 to $300 \mathrm{~K}$. For STO on LSAT, $\kappa(T)$ decreases monotonically with decreasing temperature (see Fig. 3), following the expectations of the modified Callaway model [32-34] with a concentration of defects $V_{\mathrm{O}}=$ $1.5 \%$, and $V_{\mathrm{Sr}}=1.75 \%$. However, $\kappa(T)$ of STO on DSO undergoes an important reduction below $\approx 250 \mathrm{~K}$.

Growing STO on orthorhombic DSO induces a rotation pattern $\left(a^{+} b^{-} c^{-}\right)$or $\left(a^{-} b^{+} c^{-}\right)$of the $\mathrm{TiO}_{6}$ octahedra, which is different from the case under compressive strain $\left(a^{+} b^{+} c^{0}\right)$ [28]. An out-of-phase rotation of the octahedra along the $c$ axis doubles the unit cell, and opens a phonon band gap at the new Brillouin zone edge. This could inhibit the propagation of some phonon modes and therefore reduce the thermal conductivity at room temperature. However, no change in the rotation pattern is expected to occur below 250 $\mathrm{K}$; we thus conclude that this cannot be the main reason for the change in $\kappa(T)$ reported in Fig. 3.

On the other hand, inhomogeneous relaxor ferroelectricity associated with nanopolar regions (most probably linked to 
Sr vacancies) has been reported in thin films of $\mathrm{SrTiO}_{3}$ at room temperature $[4,9,35]$. Tensile strain introduces longrange correlations among these polar domains, resulting in the development of ferroelectric domains at lower temperatures [6]. In agreement with this scenario, a strong phonon softening and a maximum in the dielectric constant (both of which are strong indications of a ferroelectric transition) were reported below $\approx 250-270 \mathrm{~K}$ in thin films of STO grown on DSO [7].

The strain fields associated with ferroelastic DWs in $\mathrm{BiFeO}_{3}, \mathrm{~Pb}\left(\mathrm{Zr}_{0.3} \mathrm{Ti}_{0.7}\right) \mathrm{O}_{3}$, and $\mathrm{PbTiO}_{3}[12,13,15]$ impose a very large thermal resistance, resulting in an important reduction of the thermal conductivity of these FE materials. Our measurements cannot discriminate between the effect of the contribution of different types of DWs, i.e., purely ferroelectric $180^{\circ}$ and ferroelastic $90^{\circ}$. The laser spot in our FDTR setup has a $1 / e^{2}$ radius of $2.85 \mu \mathrm{m}$, and therefore each measurement senses several domains and DW configurations. In any case, purely ferroelectric $180^{\circ} \mathrm{DWs}$ have been also proposed to filter transverse phonons, resulting in important reductions of $\kappa[11]$.

Therefore, the most plausible hypothesis to explain the low $\kappa$ of tensile strained STO on DSO reported in this paper, and its temperature dependence, is that phonon scattering is enhanced by DWs between regions with different polarization. Irrespective of the configuration of the domains, we could consider STO as a material in which polar regions are embedded in a matrix of nonpolar STO. Materials with this structure can be described by the effective medium model for polycrystals [36], where the ratio between the experimental thermal conductivity $\kappa$ and the intrinsic thermal conductivity of the polar or nonpolar regions $\kappa_{0}$ is

$$
\frac{\kappa_{0}}{\kappa}=1+\frac{2 R \kappa_{0}}{\phi},
$$

where $R$ is the thermal boundary resistance between polar regions. $\phi$ represents the domain size in FE phase or the average distance between the polar regions in the high-temperature relaxor ferroelectric phase. As $\kappa_{0}$ we have used the experimental thermal conductivity of STO on LSAT. Using a value of $R \approx 5 \times 10^{-9} \mathrm{~K} \mathrm{~m}^{2} \mathrm{~W}^{-1}$, the thermal boundary resistance reported for a $\mathrm{DW}$ in $\mathrm{BiFeO}_{3}$ and $\mathrm{PbTiO}_{3}[13,15]$, the average distance $\phi$ can be calculated (Fig. 4).

At room temperature, the polar regions are spaced $\approx 35-40$ $\mathrm{nm}$. Note also that Eq. (2) is valid in the diffusive regime, i.e., when the phonon mean free path $\lambda$ is smaller than the value of $\phi$. First-principles calculations of the cumulative thermal conductivity predicted a very large contribution from phonons with $\lambda<10 \mathrm{~nm}$, of about $60 \%-70 \%$ at room temperature $[37,38]$. Therefore, the use of Eq. (2) is justified, at least at room temperature.

Below $250 \mathrm{~K}$, as the ferroelectric transition becomes complete [4], the vast majority of the domains are polar and therefore now $\phi$ corresponds to the average size of these ferroelectric domains. Their size is estimated from Eq. (2) and is comparable to $\lambda$ at room temperature, although the mean free path will increase at lower temperatures and so the use of Eq. (2) becomes less justified. When $\phi<\lambda$, i.e., in the ballistic phonon transport regime, phonons can propagate

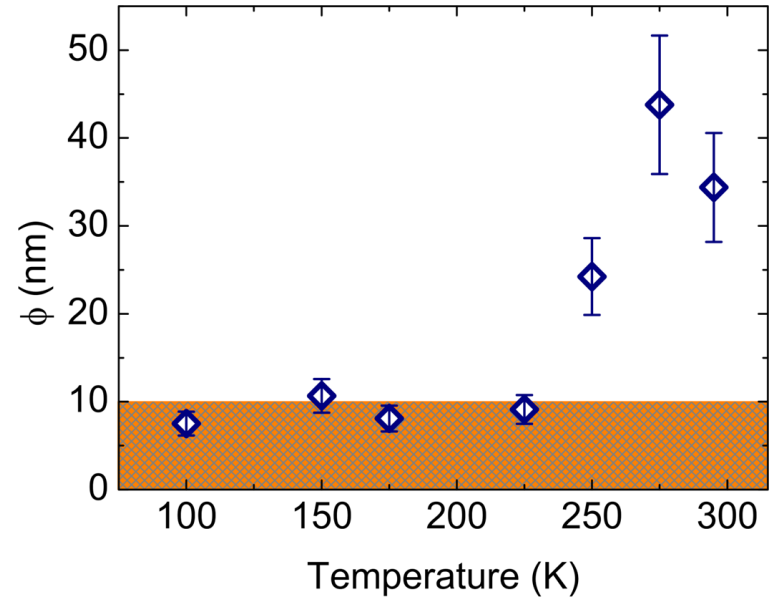

FIG. 4. Average separation between polar regions in the STO films deposited on DSO. The values are obtained from Eq. (2) and the experimental data in Fig. 3, as explained in the text. The highlighted area marks the mean free path of the phonons that contribute the most to the thermal conductivity at room temperature $[37,38]$.

without thermal resistance inside the different regions, $\kappa_{0} \rightarrow$ $\infty$, and Eq. (2) tends to $\phi=2 \kappa R$. Using the same parameters as before, $\phi \approx 7.5 \mathrm{~nm}$. This change is consistent with the increased concentration of ferroelectric domains below $\approx 250 \mathrm{~K}$.

\section{SUMMARY}

In summary, we observed a significant reduction in the room-temperature $\kappa$ of STO thin films grown under biaxial tensile strain $(\approx 1.15 \%)$, with respect to films under compressive strain and unstrained. Extending these measurements to low temperatures reveals a further reduction of $\kappa$ below $250 \mathrm{~K}$, following the behavior of a soft-phonon mode previously reported in ferroelectric STO. The $\kappa(T)$ results are consistent with the development of a macroscopic ferroelectric state below $250 \mathrm{~K}$. These results demonstrate the important role of ferroelectric domains and domain walls in the modulation of the thermal conductivity of potential thermoelectric materials, as doped STO [39].

\section{ACKNOWLEDGMENTS}

F.R. acknowledges financial support of the Ministerio de Economía y Competitividad of Spain (Project No. MAT201680762-R), and Xunta de Galicia (Centro Singular de Investigación de Galicia accreditation 2016-2019) and the European Union (European Regional Development Fund ERDF), and the European Commission through the project 734187 SPICOLOST (H2020-MSCA-RISE-2016). J.A.M. would like to acknowledge the National Science Foundation, Division of Electrical, Communication and Cyber Systems, Award No. 1901972. This work was supported by the National Natural Science Foundation of China (Grant No. 51876186), Natural Science Foundation of Zhejiang Province (Grant No. LZ19E060002), the Fundamental Research Funds for the Central Universities (K20200145), and ZJUI with W.-L. Ong as one of the Principal Supervisors. 
[1] W. Zhong and D. Vanderbilt, Phys. Rev. Lett. 74, 2587 (1995).

[2] W. Zhong and D. Vanderbilt, Phys. Rev. B 53, 5047 (1996).

[3] H. Uwe and T. Sakudo, Phys. Rev. B 13, 271 (1976).

[4] J. H. Haeni, P. Irvin, W. Chang, R. Uecker, P. Reiche, Y. L. Li, S. Choudhury, W. Tian, M. E. Hawley, B. Craigo, A. K. Tagantsev, X. Q. Pan, S. K. Streiffer, L. Q. Chen, S. W. Kirchoefer, J. Levy, and D. G. Schlom, Nature (London) 430, 758 (2004).

[5] Y. L. Li, S. Choudhury, J. H. Haeni, M. D. Biegalski, A. Vasudevarao, A. Sharan, H. Z. Ma, J. Levy, V. Gopalan, S. Trolier-McKinstry, D. G. Schlom, Q. X. Jia, and L. Q. Chen, Phys. Rev. B 73, 184112 (2006).

[6] D. Nuzhnyy, J. Petzelt, S. Kamba, P. Kužel, C. Kadlec, V. Bovtun, M. Kempa, J. Schubert, C. Brooks, and D. Schlom, Appl. Phys. Lett. 95, 232902 (2009).

[7] M. D. Biegalski, Y. Jia, D. G. Schlom, S. Trolier-McKinstry, S. K. Streiffer, V. Sherman, R. Uecker, and P. Reiche, Appl. Phys. Lett. 88, 192907 (2006).

[8] C.-H. Lee, V. Skoromets, M. D. Biegalski, S. Lei, R. Haislmaier, M. Bernhagen, R. Uecker, X. Xi, V. Gopalan, X. Martí et al., Appl. Phys. Lett. 102, 082905 (2013).

[9] H. W. Jang, A. Kumar, S. Denev, M. D. Biegalski, P. Maksymovych, C. W. Bark, C. T. Nelson, C. M. Folkman, S. H. Baek, N. Balke, C. M. Brooks, D. A. Tenne, D. G. Schlom, L. Q. Chen, X. Q. Pan, S. V. Kalinin, V. Gopalan, and C. B. Eom, Phys. Rev. Lett. 104, 197601 (2010).

[10] J. A. Seijas-Bellido, C. Escorihuela-Sayalero, M. Royo, M. P. Ljungberg, J. C. Wojdeł, J. Íñiguez, and R. Rurali, Phys. Rev. B 96, 140101(R) (2017).

[11] M. Royo, C. Escorihuela-Sayalero, J. Íñiguez, and R. Rurali, Phys. Rev. Mater. 1, 051402 (2017).

[12] J. F. Ihlefeld, B. M. Foley, D. A. Scrymgeour, J. R. Michael, B. B. McKenzie, D. L. Medlin, M. Wallace, S. TrolierMcKinstry, and P. E. Hopkins, Nano Lett. 15, 1791 (2015).

[13] P. E. Hopkins, C. Adamo, L. Ye, B. D. Huey, S. R. Lee, D. G. Schlom, and J. F. Ihlefeld, Appl. Phys. Lett. 102, 121903 (2013).

[14] S. Ning, S. C. Huberman, C. Zhang, Z. Zhang, G. Chen, and C. A. Ross, Phys. Rev. Appl. 8, 054049 (2017).

[15] E. Langenberg, D. Saha, M. E. Holtz, J. Wang, D. Bugallo, E. Ferreiro-Vila, H. Paik, I. Hanke, S. Ganschow, D. A. Muller, L.-Q. Chen, G. Catalan, N. Domingo, J. Malen, D. G. Schlom, and F. Rivadulla, Nano Lett. 19, 7901 (2019).

[16] P. Torres, J. Íñiguez, and R. Rurali, Phys. Rev. Lett. 123, 185901 (2019).

[17] A. Biswas, C.-H. Yang, R. Ramesh, and Y. H. Jeong, Prog. Surf. Sci. 92, 117 (2017).

[18] A. J. Schmidt, R. Cheaito, and M. Chiesa, Rev. Sci. Instrum. 80, 094901 (2009).

[19] J. A. Malen, K. Baheti, T. Tong, Y. Zhao, J. A. Hudgings, and A. Majumdar, J. Heat Transfer 133, 081601 (2011).

[20] C. W. Huang, Z. H. Chen, and L. Chen, J. Appl. Phys. 113, 094101 (2013).

[21] C.-S. Woo, J. H. Lee, K. Chu, B.-K. Jang, Y.-B. Kim, T. Y. Koo, P. Yang, Y. Qi, Z. Chen, L. Chen, H. C. Choi, J. H. Shim, and C.-H. Yang, Phys. Rev. B 86, 054417 (2012).
[22] S. K. Streiffer, J. A. Eastman, D. D. Fong, C. Thompson, A. Munkholm, M. V. Ramana Murty, O. Auciello, G. R. Bai, and G. B. Stephenson, Phys. Rev. Lett. 89, 067601 (2002).

[23] See Supplemental Material at http://link.aps.org/supplemental/ 10.1103/PhysRevMaterials.4.054002 for more details of the structural and transport characterization, and measurement and modeling methods, which includes Refs. [33,40-47].

[24] D.-W. Oh, J. Ravichandran, C.-W. Liang, W. Siemons, B. Jalan, C. M. Brooks, M. Huijben, D. G. Schlom, S. Stemmer, L. W. Martin et al., Appl. Phys. Lett. 98, 221904 (2011).

[25] X. Wu, J. Walter, T. Feng, J. Zhu, H. Zheng, J. F. Mitchell, N. Biškup, M. Varela, X. Ruan, C. Leighton et al., Adv. Funct. Mater. 27, 1704233 (2017).

[26] T. Ohnishi, M. Lippmaa, T. Yamamoto, S. Meguro, and H. Koinuma, Appl. Phys. Lett. 87, 241919 (2005).

[27] A. Sarantopoulos, E. Ferreiro-Vila, V. Pardo, C. Magén, M. H. Aguirre, and F. Rivadulla, Phys. Rev. Lett. 115, 166801 (2015).

[28] L. Iglesias, A. Sarantopoulos, C. Magén, and F. Rivadulla, Phys. Rev. B 95, 165138 (2017).

[29] E. Breckenfeld, A. B. Shah, and L. W. Martin, J. Mater. Chem. C 1, 8052 (2013).

[30] E. Breckenfeld, N. Bronn, J. Karthik, A. R. Damodaran, S. Lee, N. Mason, and L. W. Martin, Phys. Rev. Lett. 110, 196804 (2013).

[31] A. Beattie and G. Samara, J. Appl. Phys. 42, 2376 (1971).

[32] Z. Su, J. P. Freedman, J. H. Leach, E. A. Preble, R. F. Davis, and J. A. Malen, J. Appl. Phys. 113, 213502 (2013).

[33] F. Yang and C. Dames, Phys. Rev. B 87, 035437 (2013).

[34] D. T. Morelli and G. A. Slack, High Thermal Conductivity Materials (Springer, Berlin, 2006), pp. 37-68.

[35] A. Vasudevarao, A. Kumar, L. Tian, J. H. Haeni, Y. L. Li, C.-J. Eklund, Q. X. Jia, R. Uecker, P. Reiche, K. M. Rabe, L. Q. Chen, D. G. Schlom, and V. Gopalan, Phys. Rev. Lett. 97, 257602 (2006).

[36] C.-W. Nan and R. Birringer, Phys. Rev. B 57, 8264 (1998).

[37] L. Feng, T. Shiga, and J. Shiomi, Appl. Phys. Express 8, 071501 (2015).

[38] C. Liu, Y. Chen, and C. Dames, Phys. Rev. Appl. 11, 044002 (2019).

[39] H. Ohta, S. Kim, Y. Mune, T. Mizoguchi, K. Nomura, S. Ohta, T. Nomura, Y. Nakanishi, Y. Ikuhara, M. Hirano et al., Nat. Mater. 6, 129 (2007).

[40] E. Breckenfeld, R. Wilson, J. Karthik, A. R. Damodaran, D. G. Cahill, and L. W. Martin, Chem. Mater. 24, 331 (2012).

[41] J. Epstein, W.-L. Ong, C. J. Bettinger, and J. A. Malen, ACS Appl. Mater. Interfaces 8, 19168 (2016).

[42] A. Sarantopoulos, W.-L. Ong, J. A. Malen, and F. Rivadulla, Appl. Phys. Lett. 113, 182902 (2018).

[43] T. S. English, J. C. Duda, J. L. Smoyer, D. A. Jordan, P. M. Norris, and L. V. Zhigilei, Phys. Rev. B 85, 035438 (2012).

[44] G. A. Slack and S. Galginaitis, Phys. Rev. 133, A253 (1964).

[45] P. Klemens, Proc. Phys. Soc. London, Sect. A 68, 1113 (1955).

[46] B. Lüthi and T. Moran, Phys. Rev. B 2, 1211 (1970).

[47] R. Bell and G. Rupprecht, Phys. Rev. 129, 90 (1963). 